\title{
EXPLICIT SOLUTIONS OF SOME FRACTIONAL PARTIAL DIFFERENTIAL EQUATIONS VIA STABLE SUBORDINATORS
}

\author{
LATIFA DEBBI
}

Received 10 December 2004; Revised 5 May 2005; Accepted 10 May 2005

The aim of this work is to represent the solutions of one-dimensional fractional partial differential equations (FPDEs) of order $\left(\alpha \in \mathbb{R}_{+} \backslash \mathbb{N}\right)$ in both quasi-probabilistic and probabilistic ways. The canonical processes used are generalizations of stable Lévy processes. The fundamental solutions of the fractional equations are given as functionals of stable subordinators. The functions used generalize the functions given by the Airy integral of Sirovich (1971). As a consequence of this representation, an explicit form is given to the density of the 3/2-stable law and to the density of escaping island vicinity in vortex medium. Other connected FPDEs are also considered.

Copyright (C) 2006 Latifa Debbi. This is an open access article distributed under the Creative Commons Attribution License, which permits unrestricted use, distribution, and reproduction in any medium, provided the original work is properly cited.

\section{Introduction}

The fractional differential equations describe physical phenomena in inhomogeneous medium, such as diffusion in porous medium or with fractal geometry or with turbulence, kinematics in viscoelastic medium, relaxation processes in complex systems (viscoelastic materials, glassy materials, synthetic polymers, biopolymer), propagation of seismic waves, pollution and transport of data across the internet, for more information on this topics see $[1,12,17,18,32]$, and the references therein. The diffusion packet width, in these mediums, grows proportional to $t^{v}, v \neq 1 / 2$. When $v<1 / 2$, it is called subdiffusion, it is the case, for example, of the porous medium, and when $v>1 / 2$, it is called superdiffusion, it is the case of chaotic flows generated by vortices $[1,12,32]$. It is known that the case $t^{1 / 2}$ is the normal diffusion connected to Gaussian process. Two approaches are used to describe these diffusion processes, the first one is to consider them as a superposition of ordinary diffusions, the second one is to consider the anomaly in the microscopical scale and describe the diffusion in terms of certain random walks. This last approach gives processes generalizing the Brownian motion (Lévy processes and their

Hindawi Publishing Corporation Journal of Applied Mathematics and Stochastic Analysis Volume 2006, Article ID 93502, Pages 1-18

DOI 10.1155/JAMSA/2006/93502 
generalizations). Some properties of the anomaly are explained by the interplay of both the diffusive and ballistic behavior.

The high-order fractional partial differential equations are generalizations of the integer high-order partial differential equations. These ladders appear in the literature in connection with many physical applications, for example, heat-type equations of order three is used in the analysis of trimolecular reactions and that of order four is used in the study of the grooves developed on surfaces when obstacles are met [10]. The aim of several works on these equations is to make explicit the link between them and stochastic processes $[3,9,13,14,22,23,25-27]$ and the references therein. The major difficulty in this study is that the fundamental solution is not everywhere positive, so the stochastic representation of the solution is not always easily obtained, as it is the case for the heat equation and the Brownian motion. Krylov [16] has introduced the initial value problem

$$
\begin{gathered}
\frac{\partial u}{\partial t}=(-1)^{n+1} \frac{\partial^{2 n} u}{\partial x^{2 n}}, \quad t>0, x \in \mathbb{R}, \\
u(0, x)=f(x),
\end{gathered}
$$

for $n>1$ with $f \in L^{1}(R)$.

By Fourier's calculus, it is easy to see that the solution of (1.1) is given by

$$
u_{2 n}(t, x)=\int_{-\infty}^{+\infty} f(x+\xi) p_{2 n}(t, \xi) d \xi
$$

where $p_{2 n}(t, x)$ is the fundamental solution; that means $p_{2 n}(t, x)$ is the solution of $(1.1)$ when the initial condition is the Dirac distribution $\left(u(0, x)=\delta_{0}(x)\right) . p_{2 n}(t, x)$ can be expressed as

$$
p_{2 n}(t, x)=\frac{1}{2 \pi} \int_{-\infty}^{+\infty} \exp \left(-i x \lambda-t \lambda^{2 n}\right) d \lambda
$$

It is proven in $[13,16]$ that the function $p_{2 n}(t, x)$ is not everywhere positive when $n>1$. As a consequence, two approaches are then created. The first one is a formal probabilistic analogy with the Brownian case [9, 13, 14, 16, 22, 23, 25-27] and others. Krylov [16] has used an additive signed measure to construct a "signed probability space." The solution is then represented as $u(t, x)=\mathbb{E}\left(x+X_{t}\right)$ where $\mathbb{E}$ is the expectation and $\left\{X_{t}, t \geq 0\right\}$ is the canonical process on this space. This process is called Krylov motion or pseudoprocess and it has $p_{2 n}(t, x)$ as transition density [27]. After that, the question how to generalize the stochastic calculus to pseudoprocesses gave rise to several papers [4, 13, 22, 25-27], and so forth, where particular questions such as stochastic integral, Itô formula, first hitting time and first hitting place, Girsanov formula, stochastic differential equations, have been studied. Another formal way is to use the subordination analogy theory as in $[9,23]$.

The disadvantage of this approach is that the measure used is only finitely additive so many standard probabilistic tools cannot be applied, further the "signed probability measure" has no clear physical significance. But on the other hand, the formal stochastic calculus elaborated by this approach is useful in physics and characterizes more or less the particle and its trajectories. In [27], Nishioka represents the biharmonic pseudoprocess 
$(n=2$ in $(1.1))$ as a composition, in certain sense, of two different particles, a monopole and a dipole.

The second approach uses only probabilistic tools. In [4], Burdzy and Mądrecki gave the process connected to the fourth-order equation as a "wide limit" of a sequence of $C^{N}$-valued random processes. In this construction, they used three independent Brownian motions on a product of two probabilistic spaces, and they mentioned that they can replace two of them by stable processes. Their calculus can be applied to the fractional heat equations with differentiation order between 0 and 4: unfortunately at the end of the paper, they pointed out that this calculus leads to a paradox, further, one cannot interpret the process as the trajectories of a moving particle.

Benachour et al. [3] have used the iterated Brownian motion in the study of the fourthorder heat equation. They associated to the operator $(\partial / \partial x)^{4}$ the process $\left\{B_{\left|w_{t}\right|}, t \geq 0\right\}$, where $\left\{B_{t}, t \geq 0\right\}$ and $\left\{w_{t}, t \geq 0\right\}$ are two independent Brownian motions.

In this work, we are interested in the two approaches for the initial value problem of the fractional equations

$$
\begin{gathered}
\frac{\partial u}{\partial t}=\kappa \frac{\partial^{\alpha} u}{\partial x^{\alpha}}+c \frac{\partial u}{\partial x}, \quad t>0, x \in \mathbb{R}, \\
u(0, x)=f(x) \in L^{1}(\mathbb{R}),
\end{gathered}
$$

where $\alpha \in \mathbb{R}_{+} \backslash \mathbb{N}, \partial^{\alpha} / \partial x^{\alpha}$ is a fractional differential operator, $c$ is a real constant, and $\kappa$ is a real constant to be given later according to the differential operator taken. We note that, by a simple change of the function $u$, we can extend the study to the equation

$$
\frac{\partial u}{\partial t}=\kappa \frac{\partial^{\alpha} u}{\partial x^{\alpha}}+c \frac{\partial u}{\partial x}+c_{1} u
$$

The paper is organized as follows: after giving some basic definitions and properties in Section 2, we give in Section 3, the resolution of (1.4), the principal properties of the fundamental solution and the quasi-probabilistic approach. In Section 4, we give the probabilistic representation of the solution. To this aim, we introduce a class of functions given by Airy integral. Two subsections, at the end, are devoted to particular cases of mathematical or physical interest and to fractional equations which generalize (1.4); fractional equations in high dimension and fractional equations with more than one differential operator.

\section{Preliminaries}

In the literature, various fractional differential operators are defined, see [21, 24, 28]. The results in this paper apply to several of them, such as Riemann-Liouville operator, Nishimoto operator, and the non-selfadjoint fractional operator introduced in [5] and used in [7] to the study of stochastic fractional differential equations. 
Definition 2.1. Let $f$ be a real function. The fractional derivative of order $\alpha$ of the function $f$ in Riemann-Liouville sense, when it exists, is given by

$$
D^{\alpha} f(x)=\frac{1}{\Gamma(m-\alpha)} \frac{d^{m}}{d x^{m}} \int_{-\infty}^{x}(x-t)^{m-\alpha-1} f(t) d t, \quad m-1 \leq \alpha<m,
$$

where $m=[\alpha]+1$, and $[\alpha]$ is the integer part of $\alpha$ and $\Gamma$ is the Gamma function.

Definition 2.2. Let $f$ be an analytic function and let $z_{0}$ be a point of its domain of definition. Draw the curve $C_{+}$along the cut joining the points $z_{0}$ and $+\infty+i \mathfrak{I} z_{0}$ as $\left\{x+i \mathfrak{J} z_{0}, \mathfrak{R} z_{0}+r \leq x<+\infty\right\}^{*} \vee\left\{z_{0}+r e^{i \theta}, 0<\theta<2 \pi\right\} \vee\left\{x+i \mathfrak{J} z_{0}, \mathfrak{R} z_{0}+r \leq x<+\infty\right\}$ where the symbol $\vee$ means "followed by" and $*$ means that the curve is taken in the opposite direction. By the same way we can introduce the curve $C_{-}$along the cut joining the points $z_{0}$ and $-\infty+i \Im z_{0}$. Suppose that the function $f$ has nonbranch point inside and on the curve $C \in\left\{C_{-}, C_{+}\right\}$. The $\alpha$-fractional derivative of $f$ in the point $z_{0}$ in Nishimoto sense, if it exists, is the complex number

$$
N^{\alpha} f\left(z_{0}\right)=\frac{\Gamma(\alpha+1)}{2 \pi i} \int_{C} \frac{f(\xi)}{\left(z_{0}-\xi\right)^{\alpha+1}} d \xi
$$

In [5], the author introduced a fractional differential operator $D_{\delta}^{\alpha}$ as a generalization of the inverse of the generalized Riesz-Feller potential $[8,15]$ for $\alpha>0$.

Definition 2.3. The fractional differential $D_{\delta}^{\alpha} \varphi(x)$ is given by

$$
D_{\delta}^{\alpha} \varphi(x)=\mathscr{F}^{-1}\left\{\delta \psi_{\alpha}(\lambda) \mathscr{F}\{\varphi(x) ; \lambda\} ; x\right\},
$$

where

$$
\delta \psi_{\alpha}(\lambda)=-|\lambda|^{\alpha} e^{-i \delta(\pi / 2) \operatorname{sgn}(\lambda)},
$$

$|\delta| \leq \min \left\{\alpha-[\alpha]_{2}, 2+[\alpha]_{2}-\alpha\right\},[\alpha]_{2}$ is the largest even integer less than or equal to $\alpha$

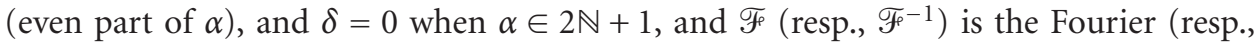
Fourier inverse) transform.

The Fourier transform and its inverse are given by

$$
\begin{gathered}
\mathscr{F}\{\phi(x) ; \lambda\}=\hat{\phi}(\lambda)=\int_{-\infty}^{+\infty} \exp (i x \lambda) \phi(x) d x, \\
\mathscr{F}^{-1}\{\phi(\lambda) ; x\}=\breve{\phi}(\lambda)=\frac{1}{2 \pi} \int_{-\infty}^{+\infty} \exp (-i x \lambda) \phi(\lambda) d \lambda .
\end{gathered}
$$

The operator $D_{\delta}^{\alpha}$ is a non-selfadjoint, closed, densely defined operator on $L^{2}(\mathbb{R})$ and it is the infinitesimal generator of, in general, a nonsymmetric and noncontraction semigroup. This operator generalizes the differentiation of high order (so (1.4) generalizes (1.1), when $\alpha$ is even $[13,16])$ and it generalizes also the fractional differential operators in $[8,11,15,20]$ for $0<\alpha \leq 2$. It is selfadjoint only when $\delta=0$, in this case, it coincides with the fractional power of the Laplacian. Evidently, when $\alpha=2$, it is the Laplacian itself. Furthermore, it is proven in [5] that when $|\delta|=2+[\alpha]_{2}-\alpha$ or $|\delta|=\alpha-[\alpha]_{2}$, it coincides 
with the Riemann-Liouville differential operator. From [15], $D_{\delta}^{\alpha}$ can be represented for $1<\alpha<2$, by

$$
D_{\delta}^{\alpha} \varphi(x)=\int_{-\infty}^{+\infty} \frac{\varphi(x+y)-\varphi(x)-y \varphi^{\prime}(x)}{|y|^{1+\alpha}}\left(M_{-}^{\delta} 1_{(-\infty, 0)}+M_{+}^{\delta} 1_{(0,+\infty)}\right) d y,
$$

and for $0<\alpha<1$, by

$$
D_{\delta}^{\alpha} \varphi(x)=\int_{-\infty}^{+\infty} \frac{\varphi(x+y)-\varphi(x)}{|y|^{1+\alpha}}\left(M_{-}^{\delta} 1_{(-\infty, 0)}+M_{+}^{\delta} 1_{(0,+\infty)}\right) d y
$$

where $M_{-}^{\delta}$ and $M_{+}^{\delta}$ are two nonnegative constants satisfying $M_{-}^{\delta}+M_{+}^{\delta}>0$ and $1_{(-\infty, 0)}$ and $1_{(0,+\infty)}$ are the indicator functions of the intervals $(-\infty, 0),(0,+\infty)$, respectively, and $\varphi$ is a smooth function for which the integrals exist, and $\varphi^{\prime}$ is its derivative. For more details about this operator see [5].

It is proven that for Definitions 2.1 and 2.2 and for smooth functions, we have the following relationship between the fractional differential operator and the Fourier transform (see [28] for Riemann-Liouville operator and see [2, 24] for Nishimoto operator):

$$
\mathscr{F}\left\{D^{\alpha} f(x) ; \lambda\right\}=(-i \lambda)^{\alpha} \mathscr{F}\{f(x) ; \lambda\} \text {. }
$$

For $0<\alpha<2$ and using the fractional operators above, we can represent the solution of (1.4) by an $\alpha$-stable process.

Definition 2.4 [30]. A real stochastic process $\left\{X_{t}, t \geq 0\right\}$ defined on a probability space $(\Omega, \mathscr{F}, \mathbf{P})$ is a Lévy process if the following conditions are satisfied:

(i) it has independent increments, that is, for any choice of $n \geq 1$ and $0 \leq t_{0} \leq t_{1} \leq$ $\cdots \leq t_{n}$, the random variables $X_{t_{0}}, X_{t_{1}}-X_{t_{0}}, \ldots, X_{t_{n}}-X_{t_{n-1}}$ are independent,

(ii) it has stationary increments, that is, for all $s, t \geq 0$ the distribution of $X_{t+s}-X_{t}$ does not depend on $t$

(iii) $X_{0}=0$ a.s.,

(iv) it is stochastically continuous; that is, $\forall t>0$ and $\varepsilon>0$,

$$
\lim _{s \rightarrow t} P\left[\left|X_{t}-X_{s}\right|>\varepsilon\right]=0,
$$

(v) it is càdlàg, that is, there is $\Omega_{0} \in \mathscr{F}$ with $P\left[\Omega_{0}\right]=1$ such that for every $\omega \in \Omega_{0}$, $X_{t}(\omega)$ is right continuous in $t \geq 0$ and has left limits in $t>0$.

The Lévy process $\left\{X_{t}, t \geq 0\right\}$ is called stable if the distribution of $X_{1}$ is stable, that is, if there are parameters $0<\alpha \leq 2, \sigma>0,-1 \leq \beta \leq 1, \mu \in \mathbb{R}$ such that the characteristic function of $X_{1}$ is given by

$$
\mathbb{E}\left[\exp i X_{1} \lambda\right]= \begin{cases}\exp \left\{-\sigma^{\alpha}|\lambda|^{\alpha}\left(1-i \beta(\operatorname{sgn} \lambda) \tan \frac{\alpha \pi}{2}\right)+i \mu \lambda\right\}, & \alpha \neq 1, \\ \exp \left\{-\sigma|\lambda|\left(1+i \beta(\operatorname{sgn} \lambda) \tan \frac{2}{\pi} \ln |\lambda|\right)+i \mu \lambda\right\}, & \alpha=1 .\end{cases}
$$

The parameters $\alpha, \sigma, \beta, \mu$ are called, respectively, stable, scale, skewness, shift parameter.

When $0<\alpha<1, \beta=1$, and $\mu \geq 0$, the stable Lévy process is called subordinator. 
Other representations of the characteristic function are also given in $[19,32]$, in particular for $\alpha \neq 1$, we have

$$
\mathbb{E}\left[\exp i X_{1} \lambda\right]=\exp \left\{-\sigma^{*}|\lambda|^{\alpha} \exp \left(-i \frac{\delta \pi}{2} \operatorname{sgn} \lambda\right)+i \mu^{*} \lambda\right\},
$$

where $|\delta| \leq \min \{\alpha, 2-\alpha\}, \sigma^{*}>0$, and $\mu^{*} \in \mathbb{R}$. We give now the extension of this definition for signed probability spaces.

Definition 2.5. Let $\left(\Omega, \mathscr{F}^{-}, \mathbf{P}^{-}\right)$be a signed probability space; that means $\mathbf{P}^{-}$is a signed measure which could be only additive and $\mathscr{F}^{-}$could be only an algebra but $\mathbf{P}^{-}(\Omega)=1$, a real pseudoprocess $\left\{X^{-}(t), t \geq 0\right\}$ is a family of $\mathscr{F}^{-}$-measurable functions.

It is clear that a pseudoprocess is a process in the classical sense when $\mathbf{P}^{-}$is a probability measure. We use the same notations as in probability: $\mathbb{E}$ denotes the "expectation" $\mathbb{E}(f)=$ $\int f d \mathbf{P}_{\alpha}^{-}$and the characteristic function is given by the formula $\mathbb{E}[\exp ($ if $\lambda)]$.

Definition 2.6. A pseudoprocess $\left\{X^{-}(t), t \geq 0\right\}$ defined on the signed probability space $\left(\Omega, \mathscr{F}^{-}, \mathbf{P}^{-}\right)$is called stable Lévy pseudoprocess if it satisfies conditions (i)-(v) in Definition 2.4 with respect to the signed probability $\mathbf{P}^{-}$and its characteristic function is given by the formula (2.11) where $\alpha \in \mathbb{R} / 2 \mathbb{N}+1,|\delta| \leq \min \left\{\alpha-[\alpha]_{2,2}+[\alpha]_{2}-\alpha\right\}, \sigma^{*}>0$, and $\mu^{*} \in \mathbb{R}$.

The notation $X_{\alpha}^{-}(1) \sim S_{\alpha}(\sigma, \beta, \mu)$ means that the characteristic function of $X_{\alpha}^{-}(1)$ is given by (2.10) where $\alpha \in \mathbb{R} / 2 \mathbb{N}+1$ and $-1<\beta<1$. The notation $\partial^{\alpha} / \partial x^{\alpha}$ is used to designate any of the fractional differential operators introduced in Definitions 2.1, 2.2, and 2.3. We take $\kappa$ equal to $-(\cos (\alpha \pi / 2))^{-1}$ for Definitions 2.1 and 2.2 and equal to $(\cos (\delta \pi / 2))^{-1}$ for Definition 2.3.

\section{Resolution of the fractional equation, some properties, and quasi-probabilistic approach}

First, we assume that the fractional differential operator in (1.4) is the Riemann-Liouville operator $\left(\partial^{\alpha} / \partial x^{\alpha}=D^{\alpha}\right)$ and applying the Fourier transform, we find the differential equation

$$
\frac{\partial \hat{u}}{\partial t}(t, \lambda)=\left(\kappa(-i \lambda)^{\alpha}+c i \lambda\right) \hat{u}(t, \lambda)
$$

where $(-i \lambda)^{\alpha}=|\lambda|^{\alpha} \exp (-i \operatorname{sgn}(\lambda)(\alpha \pi / 2))$. The solution of this differential equation taking into account the initial condition $\hat{f}$ is

$$
\widehat{u}_{\alpha}(t, \lambda)=\hat{f}(\lambda) \exp \left(\left(\kappa(-i \lambda)^{\alpha}+i c \lambda\right) t\right) .
$$

By an identical way and across the complex analysis, we obtain the same result with the Nishimoto fractional differential operator. However, for (1.4) with the fractional operator $D_{\delta}^{\alpha}$ given in Definition 2.3, we find

$$
\hat{u}_{\alpha}(t, \lambda)=\hat{f}(\lambda) \exp \left(\left(\kappa_{\delta} \psi_{\alpha}(\lambda)+i c \lambda\right) t\right)
$$


It is clear that the function $h_{\alpha}(t, \lambda)=\exp \left(\left(\kappa_{\delta} \psi_{\alpha}(\lambda)+i c \lambda\right) t\right)$ is equal to $\exp \left(\left(\kappa(-i \lambda)^{\alpha}+\right.\right.$ $i c \lambda) t$ ) when $\delta=\alpha-2-[\alpha]_{2}$ or $\delta=\alpha-[\alpha]_{2}$ conformal to Definition 2.3. Further $h_{\alpha}(t, \lambda)$ is absolutely integrable, let $p_{\alpha}(t, x)$ be its inverse Fourier transform. From the relationship $\hat{u}_{\alpha}(t, \lambda)=\hat{f}(\lambda) \hat{p}_{\alpha}(t, \lambda)$, we get

$$
u_{\alpha}(t, x)=\int_{-\infty}^{+\infty} f(\xi) p_{\alpha}(t, x-\xi) d \xi .
$$

The function $p_{\alpha}(t, x)$ is the fundamental solution of (1.4). In the sequel of this section, we take the fractional differential operator in (1.4) equal to $D_{\delta}^{\alpha}$ with general $\delta$ and we take $\kappa>0$. Let us denote the fundamental solution, in this case by $\delta p_{\alpha, c, \kappa}(t, x)$, then

$$
\delta p_{\alpha, c, \kappa}(t, x)=\frac{1}{2 \pi} \int_{-\infty}^{+\infty} \exp \left[-i \lambda(x-c t)-\kappa|\lambda|^{\alpha} t e^{-i \operatorname{sgn}(\lambda)(\delta \pi / 2)}\right] d \lambda .
$$

In the following lemma, we give some properties of the function $\delta p_{\alpha, c, \kappa}(t, x)$.

Lemma 3.1. For all $\alpha \in \mathbb{R}_{+}|\mathbb{N},| \delta \mid \leq \min \left\{\alpha-[\alpha]_{2}, 2+[\alpha]_{2}-\alpha\right\}, \kappa>0$, and $c \in \mathbb{R}$,

(i) $\int_{-\infty}^{+\infty} \delta p_{\alpha, c, \kappa}(t, x) d x=1$,

(ii) $\delta p_{\alpha, c, \kappa}(t, x)$ is real and it is not symmetric relatively to $x$ when $\delta \neq 0$ and it is not everywhere positive,

(iii) $\delta p_{\alpha, c, \kappa}(t, x)=\kappa^{-1 / \alpha} \delta p_{\alpha, \kappa^{-1 / \alpha} c, 1}\left(t, \kappa^{-1 / \alpha} x\right)$,

(iv) $\delta p_{\alpha, c, \kappa}(t, x)={ }_{\delta} p_{\alpha, 0, \kappa}(t, x-c t)$,

(v) ${ }_{-\delta} p_{\alpha, c, \kappa}(t, x)={ }_{-\delta} p_{\alpha,-c, \kappa}(t,-x)$,

(vi) $\delta p_{\alpha, 0, \kappa}(t, x)=t^{-1 / \alpha} \delta p_{\alpha, 0, \kappa}\left(1, t^{-1 / \alpha} x\right)$, (scaling property),

(vii) $\delta p_{\alpha, c, \kappa}(t, x)$ satisfies the semigroup property, or the Chapman Kolmogorov equation, that is, for $0<s<t$,

$$
\delta p_{\alpha, c, \kappa}(t+s, x)=\int_{-\infty}^{+\infty} \delta p_{\alpha, c, \kappa}(t, \xi)_{\delta} p_{\alpha, c, \kappa}(s, x-\xi) d \xi,
$$

(viii) $\delta p_{\alpha, c, \kappa}(t, \cdot) \in S^{\infty}=\left\{f \in C^{\infty}\right.$ and $\left(\partial^{\gamma} / \partial x^{\gamma}\right) f$ are bounded and tend to zero when $|x|$ tends to $\left.\infty, \forall \gamma \in \mathbb{R}_{+}\right\}$,

(ix) when $0<\alpha \leq 2$ and $\alpha \neq 1, \delta p_{\alpha, c, \kappa}(1, x)$ is the density of a stable law given by (2.11),

(x) $\delta p_{\alpha, c, \kappa}^{(l)}(1, x)=(1 / \pi) \sum_{j=1}^{n} \kappa^{(\alpha j+l) / \alpha}|x-c|^{-\alpha j-(l+1)}\left((-1)^{j+l} / j !\right) \Gamma(\alpha j+l+1) \sin j((\alpha+$ $\delta) / 2) \pi+\kappa^{(\alpha(n+1)+l) / \alpha} O\left(|x-c|^{-\alpha(n+1)-(l+1)}\right)$, when $|x|$ is large, where $\delta p_{\alpha, c, \kappa}^{(l)}(1, \cdot)$ is the derivative of order $l$ of $\delta p_{\alpha, c, \kappa}(1, \cdot)$,

(xi) $\lim _{t \rightarrow 0} \delta p_{\alpha, c, \kappa}(t, x)=\delta_{c}(x)$.

Proof. It is easy to see (i)-(ix).

(x) It is sufficient to prove this property for the function $\delta p_{\alpha, 0,1}(1, x)$ when $x>0$. In fact, using properties (iii), (iv), and (v), we get (x) for $\delta p_{\alpha, c, k}(1, x)$, and using the representation

$$
\delta p_{\alpha, c, \kappa}^{(l)}(1, x)=\frac{1}{2 \pi} \int_{-\infty}^{+\infty}(-i \lambda)^{l} \exp \left[-i \lambda(x-c)-\kappa|\lambda|^{\alpha} e^{-i(\delta \pi / 2) \operatorname{sgn}(\lambda)}\right] d \lambda,
$$

to which the same calculus also applies, we obtain the result for the derivatives. We are interested in the case $\alpha>2$, for the case $0<\alpha \leq 2$ this result can be deduced from $[19,32]$. 
The function $\delta p_{\alpha, 0,1}(1, x)$ can be written as

$$
\delta p_{\alpha, 0,1}(1, x)=\frac{1}{\pi} \mathfrak{R}\left\{\int_{0}^{+\infty} \exp \left[-i \lambda x-\lambda^{\alpha} e^{-i(\delta \pi / 2)}\right] d \lambda\right\}
$$

Let $0<r, R<\infty$, and let the curve $C_{\delta}:[r, R] \vee\left\{R e^{i \delta \theta}, 0 \leq \theta \leq \pi / 2 \alpha\right\} \vee\left\{\lambda e^{i(\delta \pi / 2 \alpha)}, r \leq \lambda \leq\right.$ $R\}^{*} \vee\left\{r e^{i \delta \theta}, 0 \leq \theta \leq \pi / 2 \alpha\right\}^{*}$, where $[r, R]$ design the segment in the real axis between $r$ and $R$, the symbol $\vee$ means followed by and $*$ means that the curve is taken in the opposite direction. By the Cauchy theorem, the integral of the function $\exp \left[-i z x-z^{\alpha} e^{-i(\delta \pi / 2)}\right]$ over $C_{\delta}$ vanishes, further the integrals over the two arcs tend to zero when $R$ tends to infinity and $r$ tends to zero, so

$$
\int_{0}^{+\infty} \exp \left[-i \lambda x-\lambda^{\alpha} e^{-i(\delta \pi / 2)}\right] d \lambda=e^{i(\pi \delta / 2 \alpha)} \int_{0}^{+\infty} \exp \left[-i \lambda x e^{i(\pi \delta / 2 \alpha)}-\lambda^{\alpha}\right] d \lambda
$$

By integrating the function $e^{i(\pi \delta / 2 \alpha)} \exp \left[-i z x e^{i(\pi \delta / 2 \alpha)}-z^{\alpha}\right]$ over the curve $C_{-1}$ when $\delta$ is positive and over $C_{1}$ when $\delta$ is negative, we get

$$
{ }_{\delta} p_{\alpha, 0,1}(1, x)=\frac{1}{\pi} \mathfrak{R}\left\{\int_{0}^{+\infty} e^{i(\pi(\delta-1) / 2 \alpha)} \exp \left[-\lambda x e^{i(\pi(\alpha+\delta-1) / 2 \alpha)}-\lambda^{\alpha} e^{-i(\pi / 2)}\right] d \lambda\right\}
$$

Making the change of variable $\xi=x \lambda$, and then expending the exponential containing $x$ in Taylor series, we find

$$
\begin{aligned}
\delta p_{\alpha, 0,1}(1, x)= & \frac{1}{\pi x} \mathfrak{R}\left\{e^{i(\pi(\delta-1) / 2 \alpha)} \int_{0}^{+\infty} \exp \left[-\xi e^{i(\pi(\alpha+\delta-1) / 2 \alpha)}-x^{-\alpha} \xi^{\alpha} e^{-i(\pi / 2)}\right] d \xi\right\} \\
= & \frac{1}{\pi x} \mathfrak{R}\left\{e^{i(\pi(\delta-1) / 2 \alpha)} \sum_{j=0}^{n} \frac{(-1)^{j}}{j !} x^{-\alpha j} e^{i(j \pi / 2)} E_{\alpha, \delta}(j)\right\} \\
& +\frac{1}{\pi x} \mathfrak{R}\left\{e^{i(\pi(\delta-1) / 2 \alpha)} \theta \frac{(-1)^{n+1}}{(n+1) !} x^{-\alpha(n+1)} e^{i((n+1) \pi / 2)} E_{\alpha, \delta}(n+1)\right\},
\end{aligned}
$$

where $E_{\alpha, \delta}(j)=\int_{0}^{+\infty} \exp \left[-\xi e^{i(\pi(\alpha+\delta-1) / 2 \alpha)}\right] \xi^{\alpha j} d \xi$ and $|\theta|<1$. By the same technique we find $E_{\alpha, \delta}(j)=\exp [-i(\pi(\alpha+\delta-1) j / 2)-i(\pi(\alpha+\delta-1) / 2 \alpha)] \Gamma(\alpha j+1), j \in \overline{1(n+1)}$. Replacing in the formula above, we find the series in (x) for $l=0$.

(xi) Let $c=0$, using the properties (vi) and (x), we prove that $\delta p_{\alpha, 0, \kappa}(t, x)$ tends to zero when $x \neq 0$ and tends to infinity when $x=0$. The case $c \neq 0$ is easily obtained thanks to the property (iv).

Proposition 3.2. For $0<\alpha<2$ and $\alpha \neq 1$, there exists a probability space $\left(\Omega, \mathscr{F}, \mathbf{P}_{\alpha}\right)$ such that the solution of (1.4) is represented by

$$
u_{\alpha}(t, x)=\mathbb{E}\left[f\left(x+X_{\alpha}(t)+c t\right)\right]
$$


where $X_{\alpha}=\left\{X_{\alpha}(t), t \geq 0\right\}$ is the canonical process on this space and which is $\alpha$-stable Lévy process totally skewed to the right (i.e., $\beta=1$ ).

For $\alpha \in(2,+\infty) \mid \mathbb{N}$, there exists a signed probability space $\left(\Omega, \mathscr{F}^{-}, \mathbf{P}_{\alpha}^{-}\right)$such that the solution of (1.4) is represented by

$$
u_{\alpha}(t, x)=\mathbb{E}\left[f\left(x+X_{\alpha}^{-}(t)+c t\right)\right]
$$

where $X_{\alpha}^{-}=\left\{X_{\alpha}^{-}(t), t \geq 0\right\}$ is the canonical pseudo stable Lévy process on this space.

Proof. We will use the same notation in the two cases.

Let $\Omega$ be the set $\{x:[0,+\infty[\rightarrow R, x(t)$ is càdlàg and $x(0)=0\}$ and $\mathscr{F}$ the algebra generated by the cylinder sets $C=\left\{x: a_{i} \leq x\left(t_{i}\right) \leq b_{i}, i=1,2, \ldots, n\right\}$, where $t_{1}<\cdots<t_{n}$ are fixed, and let the additive measure $\mathbf{P}_{\alpha}$ be defined over $C$ by

$$
\mathbf{P}_{\alpha}(C)=\int_{a_{1}}^{b_{1}} \int_{a_{2}}^{b_{2}} \cdots \int_{a_{n}}^{b_{n}} \prod_{i=1}^{i=n} \delta p_{\alpha, 0, \kappa}\left(t_{i}-t_{i-1}, x_{i}-x_{i-1}\right) d x_{i}
$$

where $t_{0}=0$ and $x_{0}=0$.

For $0<\alpha<2$ and $\alpha \neq 1, \mathbf{P}_{\alpha}$ can be extended to a positive measure denoted also by $\mathbf{P}_{\alpha}$, over a $\sigma$-algebra $\mathscr{F}_{\text {, further }} h_{\alpha}(t, \lambda)$ is definite positive, $h_{\alpha}(t, 0)=1$ and it is continuous, so by Bochner theorem, $\mathbf{P}_{\alpha}$ is a probability measure. We take $\left\{X_{\alpha}(t), t \geq 0\right\}$ to be the canonical process on probability space $\left(\Omega, \mathscr{F}, \mathbf{P}_{\alpha}\right)$.

For $\alpha \in(2,+\infty) \mid \mathbb{N}$, the function $p_{\alpha}(t, x)$ is not everywhere positive; Lemma 3.1. In this case, we consider the signed space $\left(\Omega, \mathscr{F}, \mathbf{P}_{\alpha}\right)$, and the canonical pseudoprocess $\left\{X_{\alpha}(t)\right.$, $t \geq 0\}$ as it is defined in [13]. It is easy to see that

$$
\mathbf{P}_{\alpha}\left(X_{t_{1}}<x_{1}, X_{t_{2}}<x_{2}, \ldots, X_{t_{n}}<x_{n}\right)=\int_{-\infty}^{x_{1}} \int_{-\infty}^{x_{2}} \cdots \int_{-\infty}^{x_{n}} \prod_{i=1}^{i=n} \delta p_{\alpha, 0, \kappa}\left(t_{i}-t_{i-1}, x_{i}-x_{i-1}\right) d x_{i}
$$

and for $0 \leq t_{0}<t_{1}<\cdots<t_{n}$,

$$
\mathbb{E}\left[\exp i \sum_{j=1}^{n} \lambda_{j}\left(X_{\alpha}\left(t_{j}\right)-X_{\alpha}\left(t_{j-1}\right)\right)\right]=\prod_{j=1}^{n} \mathbb{E}\left[\exp i \lambda_{j}\left(X_{\alpha}\left(t_{j}\right)-X_{\alpha}\left(t_{j-1}\right)\right)\right]
$$

hence $X_{\alpha}$ has independent stationary increments. To prove that $X_{\alpha}$ is stochastically continuous, it is sufficient to prove that $\mathbf{P}_{\alpha}\left[\left|X_{\alpha}\right|>\varepsilon\right] \rightarrow 0$. This follows from property (xi) in Lemma 3.1. The representations (3.12) and (3.13) rise from (3.4) and the property (iv) of Lemma 3.1.

The signed probability $\mathbf{P}_{\alpha}$ and the pseudo stable Lévy process $X_{\alpha}$ have also the following properties.

Corollary 3.3. (i) $\mathbf{P}_{\alpha}$ has unbounded total variation on paths $x(t)$,

(ii) $X_{\alpha}$ is a selfsimilar pseudoprocess, 
(iii) $X_{\alpha}$ is an infinitely divisible pseudoprocess and its spectral representation (generalized Lévy Khinchine canonical formula) when $\delta=\alpha-[\alpha]_{2}$ or $\delta=\alpha-[\alpha]_{2}-2$ is

$$
h_{\alpha}(t, \lambda)=\exp \left[Q \int_{0}^{+\infty} \exp \left(i x \lambda|\lambda|^{[\alpha]_{2} /\left(\alpha-[\alpha]_{2}\right)}-1-\frac{i x \lambda|\lambda|^{[\alpha]_{2} /\left(\alpha-[\alpha]_{2}\right)}}{1+x^{2}}\right) \frac{1}{|x|^{1+\alpha-[\alpha]_{2}}} d x\right]
$$

where $Q$ is a real nonnegative number,

(iv) let $f$ be a deterministic function, a formal integral of $f$ with respect to the pseudoprocess $Y_{\alpha}=\left\{Y_{\alpha}(t)=X_{\alpha}(t)+c t, t \geq 0\right\}$ is the pseudo stable Lévy process $\int_{0}^{t} f(s) d Y_{\alpha} \sim$ $S_{\alpha}\left(\left(\int_{0}^{t}|f(s)|^{\alpha} d s\right)^{1 / \alpha}, \int_{0}^{t}|f(s)|^{\langle\alpha\rangle} d s / \int_{0}^{t}|f(s)|^{\alpha} d s, c \int_{0}^{t} f(s d s)\right)$, where $x^{\langle\alpha\rangle}=\operatorname{sgn}(x)|x|^{\alpha}$. This definition is a formal extension of that given in [29] for stable processes.

\section{Representation of the solution using probabilistic tools}

In this section we take $\delta=\alpha-[\alpha]_{2}$ or $\delta=2-\alpha+[\alpha]_{2}$. First, we introduce certain functions that generalize Airy function. These functions are more general than those given in [6], and simpler and more general than those given in [31]. The functions in [31] are given for integral exponents, $k \in \mathbb{N}$, and they are represented by integrals over $k+1$ curves.

Lemma 4.1. Let $\beta>1$ and $n \in \mathbb{N}$ be fixed. The function $\psi_{\beta, n}$ defined on $\mathbb{R}$ by

$$
\psi_{\beta, n}(x)=\frac{1}{\pi} \int_{0}^{+\infty} \exp \left(-i\left(x \lambda+(-1)^{n} \lambda^{\beta}\right)\right) d \lambda
$$

is well defined and it is infinitely differentiable. Further when $\beta=2 m+1$ and $n=m+1$, the real part of this function, $\mathfrak{R}\left\{\psi_{2 m+1, m+1}(x)\right\}$, is solution of the equation

$$
(2 m+1) v^{(2 m)}-x v=0,
$$

where $v^{(2 m)}$ is the derivative of order $2 m$ of the function $v$.

Proof. For fixed $x$, consider the analytic function

$$
f_{\beta, n}(x, z)=\exp \left(-i\left(x z+(-1)^{n} z^{\beta}\right)\right), \quad z \in \mathbb{C},
$$

with $z^{\beta}$ is a branch of the multiform exponent function. Let the curve $C_{\beta, n}$ given by $C_{\beta, n}=$ $[r, R] \vee\left\{R \exp \left(i(-1)^{n+1} \theta\right), \quad \theta \in(0, \pi / 2 \beta)\right\} \vee\left\{\lambda \exp \left(i\left((-1)^{n+1} \pi / 2 \beta\right)\right), \quad \lambda \in(r, R)\right\}^{*} \vee$ $\left\{r \exp \left(i(-1)^{n+1} \theta\right), \theta \in(0, \pi / 2 \beta)\right\}^{*}$. By Cauchy theorem, we have

$$
\oint_{C_{\beta, n}} f_{\beta, n}(x, z) d z=0
$$


On the other hand,

$$
\begin{aligned}
\oint_{C_{\beta, n}} f_{\beta, n}(x, z) d z \\
=\int_{r}^{R} \exp \left(i\left(-x \lambda+(-1)^{n+1} \lambda^{\beta}\right)\right) d \lambda \\
\quad+i(-1)^{n+1} \int_{0}^{\pi / 2 \beta} \exp \left(-i R e^{i(-1)^{n+1} \theta} x+i(-1)^{n+1} R^{\beta} e^{i(-1)^{n+1} \beta \theta}\right) R e^{i(-1)^{n+1} \theta} d \theta \\
\quad-\int_{r}^{R} \exp \left(-i x \lambda e^{i(-1)^{n+1}(\pi / 2 \beta)}-\lambda^{\beta}\right) e^{i(-1)^{n+1}(\pi / 2 \beta)} d \lambda \\
\quad-i(-1)^{n+1} \int_{0}^{\pi / 2 \beta} \exp \left(-i r e^{i(-1)^{n+1} \theta} x+i(-1)^{n+1} r^{\beta} e^{i(-1)^{n+1} \beta \theta}\right) r e^{i(-1)^{n+1} \theta} d \theta .
\end{aligned}
$$

It is easy to see that the last integral tends to zero. Further,

$$
\begin{aligned}
\left|I_{R}\right| & =\left|\int_{0}^{\pi / 2 \beta} \exp \left(i\left(-x R e^{i(-1)^{n+1} \theta}+(-1)^{n+1} R^{\beta} e^{i(-1)^{n+1} \beta \theta}\right)\right) i R e^{\left(i(-1)^{n+1} \theta\right)} d \theta\right| \\
& \left.\leq R \int_{0}^{\pi / 2 \beta} \exp (-1)^{n+1} x R \sin \theta-R^{\beta} \sin \beta \theta\right) d \theta .
\end{aligned}
$$

For $R>(2|x|)^{(\beta-1)^{-1}}$, we have

$$
\left|I_{R}\right| \leq R \int_{0}^{\pi / 2} \exp \left[-\frac{R^{\beta} \sin \theta}{2}\right] d \theta
$$

hence $I_{R} \underset{R \rightarrow+\infty}{\longrightarrow}$, consequently

$$
\int_{0}^{+\infty} \exp \left(-i\left(x \lambda+(-1)^{n} \lambda^{\beta}\right)\right) d \lambda=e^{i(-1)^{n+1}(\pi / 2 \beta)} \int_{0}^{\infty} \exp \left(-i x \lambda e^{i(-1)^{n+1}(\pi / 2 \beta)}-\lambda^{\beta}\right) d \lambda .
$$

The function $k_{\beta, n}(x, \lambda)=e^{i(-1)^{n+1}(\pi / 2 \beta)} \exp \left(-i x \lambda e^{i(-1)^{n+1}(\pi / 2 \beta)}-\lambda^{\beta}\right)$ is absolutely integrable with respect to $\lambda$ because it is locally integrable and for $\lambda>(2|x|)^{(\beta-1)^{-1}}, \mid k_{\beta, n} \times$ $(x, \lambda) \mid=\exp \left[(-1)^{n+1} x \lambda \sin (\pi / 2 \beta)-\lambda^{\beta}\right] \leq \exp \left[-\lambda^{\beta} / 2\right]$.

Here and in the sequel, we consider the integral on the right-hand side of (4.8) in the Lebesgue sense.

The function $\psi_{\beta, n}(x)$ is infinitely differentiable because, for all $l \in \mathbb{N}$,

$$
\int_{0}^{+\infty} \lambda^{l} \exp \left[(-1)^{n+1} x \lambda \sin \frac{\pi}{2 \beta}-\lambda^{\beta}\right] d \lambda<\infty
$$


and for a point $x$, let $D>|x|$, the function $\left|\left(-i \lambda e^{i(-1)^{n+1}(\pi / 2 \beta)}\right)^{l} k_{\beta, n}(x, \lambda)\right|$ is lower bounded by the integrable function $g(\lambda)$ given by

$$
g(\lambda)= \begin{cases}\lambda^{l} \exp \left(-\frac{\lambda^{\beta}}{2}\right), & \lambda>(2 D)^{(\beta-1)^{-1}}, \\ \lambda^{l} \sup _{x \in \overline{]-D, D}}\left|k_{\beta, n}(x, \lambda)\right|, & \lambda \leq(2 D)^{(\beta-1)^{-1}} .\end{cases}
$$

Furthermore

$$
\psi_{\beta, n}^{(l)}(x)=\frac{1}{\pi} e^{(i \pi / 2)\left((-1)^{n+1}(l+1) / \beta-l\right)} \int_{0}^{+\infty} \lambda^{l} \exp \left[-i x \lambda \exp \left(i \frac{(-1)^{n+1} \pi}{2 \beta}\right)-\lambda^{\beta}\right] d \lambda .
$$

When $\beta=2 m+1$ and $n=m+1$, we have from (4.11)

$$
\mathfrak{R}\left\{\psi_{2 m+1, m+1}^{(2 m)}(x)\right\}=\frac{1}{\pi} \mathfrak{R}\left\{i \int_{0}^{+\infty} \lambda^{2 m} \exp \left[-i x \lambda \exp \left(i \frac{(-1)^{m} \pi}{2(2 m+1)}\right)-\lambda^{2 m+1}\right] d \lambda\right\} .
$$

On the other hand, we have

$$
\begin{aligned}
x \mathfrak{R}\{ & \left.\psi_{2 m+1, m+1}(x)\right\} \\
& =\frac{1}{\pi} \mathfrak{R}\left\{e^{i\left((-1)^{m} \pi / 2(2 m+1)\right)} \int_{0}^{+\infty} x \exp \left[-i x \lambda e^{i\left((-1)^{m} \pi / 2(2 m+1)\right)}\right] \exp \left(-\lambda^{2 m+1}\right) d \lambda\right\} \\
& =\frac{1}{\pi} \mathfrak{R}\left\{i \int_{0}^{+\infty} d\left(\exp \left[-i x \lambda e^{i\left((-1)^{m} \pi / 2(2 m+1)\right)}\right]\right) \exp \left(-\lambda^{2 m+1}\right)\right\} .
\end{aligned}
$$

Integrating by parts, we obtain

$$
\begin{aligned}
x \mathfrak{R}\{ & \left.\psi_{2 m+1, m+1}(x)\right\} \\
& =\frac{1}{\pi} \mathfrak{R}\left\{i+i(2 m+1) \int_{0}^{+\infty} \lambda^{2 m} \exp \left[-i x \lambda e^{i\left((-1)^{m} \pi / 2(2 m+1)\right)}\right] \exp \left(-\lambda^{2 m+1}\right) d \lambda\right\} \\
& =(2 m+1) \mathfrak{R}\left\{\psi_{2 m+1, m+1}^{(2 m)}(x)\right\} .
\end{aligned}
$$

Remark 4.2. For $\beta=3$ and $n=2, \psi_{3,2}(x)=(1 / \pi) \Re \int_{0}^{+\infty} \exp \left(-i\left(x \lambda+\lambda^{3}\right)\right) d \lambda$ is the Airy function.

Remark 4.3. The particular case $\beta=2 m+1$ and $n=m+1$ has special interest in this work as we will see below.

Theorem 4.4. Let $\alpha \in(2,+\infty) \mid \mathbb{N}$ such that its integer part is even $\left([\alpha]=[\alpha]_{2}\right)$, then there exists a probability space $\left(\Omega, \mathscr{F}_{F}, \mathbf{P}\right)$ and a stable subordinator $\left\{X_{\alpha-[\alpha]_{2}}(t), t \geq 0\right\}$ with $X_{\alpha-[\alpha]_{2}}(1) \sim S_{\alpha-[\alpha]_{2}}(1,1,0)$ such that the solution of $(1.4)$ is represented by

$$
u_{\alpha}(t, x)=\int_{-\infty}^{+\infty} f(\xi) p_{\alpha}(t, x-\xi) d \xi
$$


where

$$
\begin{gathered}
p_{\alpha}(t, x)=\mathbb{E}\left[\Phi_{\alpha}^{1}\left(x-c t, X_{\alpha-[\alpha]_{2}}(t)\right)\right], \quad t>0, \\
\Phi_{\alpha}^{1}(x, y)=y^{-\left(\alpha-[\alpha]_{2}\right) / \alpha} \psi_{\alpha /\left(\alpha-[\alpha]_{2}\right), 1}\left(x y^{-\left(\alpha-[\alpha]_{2}\right) / \alpha}\right), \quad y>0,
\end{gathered}
$$

with $\psi_{\alpha /\left(\alpha-[\alpha]_{2}\right), 1}$ is given by (4.1).

Proof. First we consider the case $c=0$. From (3.5), we have

$$
p_{\alpha}(t, x)=\frac{1}{2 \pi} \int_{-\infty}^{+\infty} \exp \left[-i \lambda x-|\lambda|^{\alpha} t\left(1-i \operatorname{sgn}(\lambda) \tan \frac{\left(\alpha-[\alpha]_{2}\right) \pi}{2}\right)\right] d \lambda,
$$

then we can write

$$
h_{\alpha}(t, \lambda)=h_{\alpha-[\alpha]_{2}}\left(t, \lambda|\lambda|^{[\alpha]_{2} /\left(\alpha-[\alpha]_{2}\right)}\right)=\mathbb{E}\left(\exp i \lambda|\lambda|^{[\alpha]_{2} /\left(\alpha-[\alpha]_{2}\right)} X_{\alpha-[\alpha]_{2}}(t)\right),
$$

where $h_{\alpha-[\alpha]_{2}}(t, x)$ is the characteristic function of the $\left(\alpha-[\alpha]_{2}\right)$-stable subordinator $X_{\alpha-[\alpha]_{2}}$ in time $t$; Proposition 3.2. The fundamental solution $p_{\alpha}(t, x)$ can be given by

$$
\begin{aligned}
p_{\alpha}(t, x) & =\frac{1}{2 \pi} \int_{-\infty}^{+\infty} e^{-i x \lambda} h_{\alpha-[\alpha]_{2}}\left(t, \lambda|\lambda|^{[\alpha]_{2} /\left(\alpha-[\alpha]_{2}\right)}\right) d \lambda \\
& =\frac{1}{2 \pi} \int_{-\infty}^{+\infty} e^{-i x \lambda}\left(\int_{0}^{+\infty} \exp \left[i \lambda|\lambda|^{[\alpha]_{2} /\left(\alpha-[\alpha]_{2}\right)} y\right] p_{\alpha-[\alpha]_{2}}(t, y) d y\right) d \lambda \\
& =\frac{1}{\pi} \mathfrak{R}\left\{\int_{0}^{+\infty} e^{-i x \lambda}\left(\int_{0}^{+\infty} \exp \left[i \lambda^{\alpha /\left(\alpha-[\alpha]_{2}\right)} y\right] p_{\alpha-[\alpha]_{2}}(t, y) d y\right) d \lambda\right\} \\
& =\lim _{M \rightarrow+\infty} \mathfrak{R}\left\{\int_{0}^{+\infty}\left(\frac{1}{\pi} \int_{0}^{M} \exp \left[-i\left(x \lambda-\lambda^{\alpha /\left(\alpha-[\alpha]_{2}\right)} y\right)\right] d \lambda\right) p_{\alpha-[\alpha]_{2}}(t, y) d y\right\} \\
& =\lim _{M \rightarrow+\infty} \mathfrak{R}\left\{\int_{0}^{+\infty} \Phi_{\alpha, M}(x, y) p_{\alpha-[\alpha]_{2}}(t, y) d y\right\},
\end{aligned}
$$

where $M \in \mathbb{N}$ and $\Phi_{\alpha, M}(x, y)=(1 / \pi) \int_{0}^{M} \exp \left[-i\left(x \lambda-\lambda^{\alpha /\left(\alpha-[\alpha]_{2}\right)} y\right)\right] d \lambda$. It is clear that for fixed $y$ and $x$, the sequence of functions $\Phi_{\alpha, M}(x, y)$ tends to the function $\Phi_{\alpha}^{1}(x, y)=$ $y^{-\left(\alpha-[\alpha]_{2}\right) / \alpha} \psi_{\alpha /\left(\alpha-[\alpha]_{2}\right), 1}\left(x y^{-\left(\alpha-[\alpha]_{2}\right) / \alpha}\right)$ when $M$ tends to infinity, where the function $\psi_{\alpha /\left(\alpha-[\alpha]_{2}\right), 1}$ is given in Lemma 4.1. Further, we can apply Lebesgue theorem. In fact, using (4.8), we obtain $\left|\Phi_{\alpha, M}(x, y)\right| \leq(1 / \pi) \int_{0}^{+\infty} \exp \left[x \lambda \sin \left(\left(\alpha-[\alpha]_{2}\right) \pi / 2 \alpha\right)-\lambda^{\alpha /\left(\alpha-[\alpha]_{2}\right)} y\right] d \lambda$, and by Fubini's theorem, we get

$$
\begin{aligned}
& \frac{1}{\pi} \int_{0}^{+\infty} p_{\alpha-[\alpha]_{2}}(t, y)\left(\int_{0}^{+\infty} \exp \left[x \lambda \sin \frac{\left(\alpha-[\alpha]_{2}\right) \pi}{2 \alpha}-\lambda^{\alpha /\left(\alpha-[\alpha]_{2}\right)} y\right] d \lambda\right) d y \\
& \quad \leq \frac{1}{\pi} \int_{0}^{+\infty}\left[\exp \left(x \lambda \sin \frac{\left(\alpha-[\alpha]_{2}\right) \pi}{2 \alpha}\right)\left(\int_{0}^{+\infty} \exp \left(-y \lambda^{\alpha /\left(\alpha-[\alpha]_{2}\right)}\right) p_{\alpha-[\alpha]_{2}}(t, y) d y\right)\right] d \lambda .
\end{aligned}
$$


But the second integral on the right-hand side of the above inequality is the Laplace transform of the subordinator $X_{\alpha-[\alpha]_{2}}(t)$ in time $t$, so it is equal to $\exp \left(-\left(\cos \left(\left(\alpha-[\alpha]_{2}\right) \pi / 2\right)\right)^{-1}\right.$ $\left.t \lambda^{\alpha}\right)$ [29]. Hence

$$
\begin{aligned}
& \int_{0}^{+\infty}\left|\Phi_{\alpha, M}(x, y)\right| p_{\alpha-[\alpha]_{2}}(t, y) d y \\
& \quad \leq \frac{1}{\pi} \int_{0}^{+\infty} \exp \left[x \lambda \sin \frac{\left(\alpha-[\alpha]_{2}\right) \pi}{2 \alpha}-\left(\cos \frac{\left(\alpha-[\alpha]_{2}\right) \pi}{2}\right)^{-1} t \lambda^{\alpha}\right] d \lambda,
\end{aligned}
$$

by the same technique as above, we can see that the integral on the right-hand side in this last inequality is finite. Therefore

$$
\begin{aligned}
p_{\alpha}(t, x) & =\int_{0}^{+\infty} p_{\alpha-[\alpha]_{2}}(t, y) \mathfrak{R}\left\{\frac{1}{\pi} \int_{0}^{+\infty} \exp \left[-i\left(x \lambda-\lambda^{\alpha /\left(\alpha-[\alpha]_{2}\right)} y\right)\right] d \lambda\right\} d y \\
& =\int_{0}^{+\infty} p_{\alpha-[\alpha]_{2}}(t, y)\left[y^{-\left(\alpha-[\alpha]_{2}\right) / \alpha} \psi_{\alpha /\left(\alpha-[\alpha]_{2}\right), 1}\left(x y^{-\left(\alpha-[\alpha]_{2}\right) / \alpha}\right)\right] d y \\
& =\mathbb{E}\left[\Phi_{\alpha}^{1}\left(x, X_{\alpha-[\alpha]_{2}}(t)\right)\right] .
\end{aligned}
$$

When $c \neq 0$, we use the property (iv), we find (4.16).

Theorem 4.5. Let $\alpha=n+1 / 2$ with $n \in \mathbb{N}$, then there exist a probability space $\left(\Omega, \mathscr{F}, \mathbf{P}_{\alpha}\right)$ and a stable Lévy motion $\left\{X_{1 / 2}(t), t \geq 0\right\}$ with $X_{1 / 2}(1) \sim S_{1 / 2}(1,1,0)$ defined on it such that the solution of (1.4) is represented by

$$
u_{n+1 / 2}(t, x)=\int_{-\infty}^{+\infty} f(\xi) p_{n+1 / 2}(t, x-\xi) d \xi
$$

where

$$
\begin{gathered}
p_{n+1 / 2}(t, x)=\mathbb{E}\left[\Phi_{n+1 / 2}\left(x-c t, X_{1 / 2}(t)\right)\right], \quad t>0, \\
\Phi_{n+1 / 2}(x, y)=y^{-1 /(2 n+1)} \psi_{2 n+1, n+1}\left(x y^{-1 /(2 n+1)}\right), \quad y>0,
\end{gathered}
$$

with $\psi_{2 n+1, n+1}$ is given by (4.1).

Proof. Thanks to property (iv) in Lemma 3.1, we can take without restriction $c=0$.

When $n=0$, we have

$$
\frac{\partial u}{\partial t}=-\sqrt{2} \frac{\partial^{1 / 2} u}{\partial x^{1 / 2}}, \quad u(0, x)=f(x) .
$$

By Proposition 3.2, there exist a probability space $\left(\Omega, \mathscr{F}, \mathbf{P}_{\alpha}\right)$ and a Lévy motion $X_{1 / 2}=$ $\left\{X_{1 / 2}(t), t \geq 0\right\}\left(X_{1 / 2}(1) \sim S_{1 / 2}(1,1,0)\right)$ such that

$$
h_{1 / 2}(t, \lambda)=\mathbb{E} \exp \left(i \lambda X_{1 / 2}(t)\right) .
$$

It is known that the density of $X_{1 / 2}(t)$ is given by

$$
p_{1 / 2}(t, x)= \begin{cases}\frac{t}{2 \sqrt{\pi}} x^{-3 / 2} e^{-t^{2} / 4 x}, & x>0, \\ 0, & x \leq 0 .\end{cases}
$$


For $n>0$ and similarly in the proof of Theorem 4.4 , we regard $h_{n+1 / 2}(t, \lambda)$ as

$$
h_{n+1 / 2}(t, \lambda)=h_{1 / 2}\left(t,(-1)^{n} \lambda^{2 n+1}\right)=\mathbb{E}\left(\exp i(-1)^{n} \lambda^{2 n+1} X_{1 / 2}(t)\right),
$$

where $h_{1 / 2}(t, x)$ is the characteristic function of the Lévy motion $X_{1 / 2}=\left\{X_{1 / 2}(t), t \geq 0\right\}$ in time $t$. The fundamental solution $p_{\alpha}(t, x)$ is given by

$$
\begin{aligned}
p_{n+1 / 2}(t, x) & =\frac{1}{\pi} \mathfrak{R}\left\{\int_{0}^{+\infty} e^{-i x \lambda}\left(\int_{0}^{+\infty} \exp \left[i(-1)^{n} \lambda^{2 n+1} y\right] p_{1 / 2}(t, y) d y\right) d \lambda\right\} \\
& =\lim _{M \rightarrow+\infty} \mathfrak{R}\left\{\int_{0}^{+\infty}\left(\frac{1}{\pi} \int_{0}^{M} \exp \left[-i\left(x \lambda+(-1)^{n+1} \lambda^{2 n+1} y\right)\right] d \lambda\right) p_{1 / 2}(t, y) d y\right\} \\
& =\lim _{M \rightarrow+\infty} \mathfrak{R}\left\{\int_{0}^{+\infty} \Phi_{n, M}(x, y) p_{1 / 2}(t, y) d y\right\},
\end{aligned}
$$

where $\Phi_{n, M}(x, y)=(1 / \pi) \int_{0}^{M} \exp \left[-i\left(x \lambda+(-1)^{n+1} \lambda^{2 n+1} y\right)\right] d \lambda$. It is easy to see that, for fixed $x$ and $y, \Phi_{n, M}(x, y)$ tends to $\Phi_{n}(x, y)=y^{-1 /(2 n+1)} \psi_{2 n+1, n+1}\left(x y^{-1 /(2 n+1)}\right)$ when $M$ tends to infinity, where $\psi_{2 n+1, n+1}$ is given in Lemma 4.1. Using the following estimation:

$$
\begin{aligned}
& \frac{1}{\pi} \int_{0}^{+\infty}\left[\exp \left((-1)^{n} x \lambda \sin \frac{\pi}{2(2 n+1)}\right)\left(\int_{0}^{+\infty} \exp \left(-y \lambda^{2 n+1}\right) p_{1 / 2}(t, y) d y\right)\right] d \lambda \\
& \leq \frac{1}{\pi} \int_{0}^{+\infty} \exp \left[(-1)^{n} x \lambda \sin \frac{\pi}{2(2 n+1)}-\sqrt{2} t \lambda^{(2 n+1) / 2}\right] d \lambda,
\end{aligned}
$$

and the same technique in Theorem 4.4, we get

$$
\begin{aligned}
p_{n+1 / 2}(t, x) & =\int_{0}^{+\infty} p_{1 / 2}(t, y) \mathfrak{R}\left\{\frac{1}{\pi} \int_{0}^{+\infty} \exp \left[-i\left(x \lambda+(-1)^{n+1} \lambda^{2 n+1} y\right)\right] d \lambda\right\} d y \\
& =\int_{0}^{+\infty} p_{1 / 2}(t, y) y^{-1 /(2 n+1)} \psi_{2 n+1, n+1}\left(x y^{-1 /(2 n+1)}\right) d y \\
& =\mathbb{E}\left[\Phi_{n+1 / 2}\left(x, X_{1 / 2}(t)\right)\right] .
\end{aligned}
$$

4.1. Some special cases. (i) For $\alpha=3 / 2$, we have

$$
p_{3 / 2}(t, x)=\mathbb{E}\left[\left(X_{1 / 2}(t)\right)^{-1 / 3} A i\left((x-c t)\left(X_{1 / 2}(t)\right)^{-1 / 3}\right)\right]
$$

The function $p_{3 / 2}(1, x)$ is the density of the $3 / 2$-stable law,

(ii) $\alpha=5 / 2$ and $c=0$, we have

$$
\begin{gathered}
h_{5 / 2}(t, \lambda)=\exp \left(-t|\lambda|^{5 / 2}(1-i \operatorname{sgn} \lambda)\right)=\mathbb{E} \exp \left(i \lambda^{5} X_{1 / 2}(t)\right), \\
p_{5 / 2}(t, x)=\mathbb{E}\left[\left(X_{1 / 2}(t)\right)^{-1 / 5} \Phi_{5 / 2}\left(x\left(X_{1 / 2}(t)\right)^{-1 / 5}\right)\right] .
\end{gathered}
$$

The function $p_{5 / 2}(t, x)$ can represent the probability density of escaping the island vicinity after being in its neighborhood for a time $t$, in vortex medium [18]. 
4.2. Some other PDEs connected with (1.4). In this subsection, we consider two equations connected with (1.4). The first one corresponds to the vectorial case $\left(x \in R^{m}\right)$. In the second one the variable $x$ is real and we use a sum of fractional differential operators.

(1) Let us consider the equation

$$
\begin{aligned}
\frac{\partial u}{\partial t}=\kappa \Delta_{\alpha} u, \quad t & \geq 0, \quad x \in R^{m}, \\
u(0, x) & =f(x),
\end{aligned}
$$

where $\Delta_{\alpha}=\partial^{\alpha} / \partial x_{1}^{\alpha}+\partial^{\alpha} / \partial x_{2}^{\alpha}+\cdots+\partial^{\alpha} / \partial x_{m}^{\alpha}$, and $\partial^{\alpha} / \partial x_{i}^{\alpha}$ is given above. The solution is

$$
u_{\alpha}(t, x)=\int_{-\infty}^{+\infty} \cdots \int_{-\infty}^{+\infty} f(x+\xi) P_{\alpha}(t, \xi) d \xi
$$

where $P_{\alpha}(t, x)=\prod_{j=1}^{m} p_{\alpha}\left(t, x_{j}\right)$ and $p_{\alpha}\left(t, x_{j}\right)$ are given by (4.16) or (4.24) taking $c=0$.

(2) The second one is

$$
\frac{\partial u}{\partial t}=\sum_{k=0}^{r} \kappa_{k} \frac{\partial^{\alpha_{k}} u}{\partial x^{\alpha_{k}}}, \quad u(0, x)=f(x),
$$

where $r \in N, \alpha=\left(\alpha_{1}, \alpha_{2}, \ldots, \alpha_{r}\right) \in(\mathbb{R}+/ \mathbb{N})^{r}$, and $\kappa_{k}$ depends on $\alpha_{k}$ and it is given as above. Then the solution $u_{\alpha_{1}, \alpha_{2}, \ldots, \alpha_{r}}(t, x)$ is equal to $f * p_{\alpha_{1}}(t, x) * p_{\alpha_{2}}(t, x) *$ $\cdots * p_{\alpha_{r}}(t, x)$, where $p_{\alpha_{i}}(t, x)$ are given by (4.16) or (4.24) taking $c=0$.

Open question. The case $[\alpha]$ is odd and $\alpha-[\alpha] \neq 1 / 2$ is still open. In this case, it is easy to see that we can represent the characteristic function $h_{\alpha}(t, \lambda)$ by the characteristic function of a $\gamma$-stable law with $1<\gamma<2$, however if we try to represent the density $p_{\alpha}(t, x)$ by this technique of subordination or by replacing $p_{\gamma}(t, x)$ by $p_{1 / \gamma}(t, x)$ we encounter divergent integrals.

\section{Acknowledgments}

I would like to thank Professor R. Dalang for pointing out this question to me, and Professor P. Y. Gaillard for the conversation about Lemma 4.1.

\section{References}

[1] V. V. Anh and N. N. Leonenko, Spectral analysis of fractional kinetic equations with random data, Journal of Statistical Physics 104 (2001), no. 5-6, 1349-1387.

[2] F. Ben Adda, Dérivation d'ordre réel, Etude, Application et Interprétation géométrique, Thèse, Universitè Paris VI, Paris, 1997.

[3] S. Benachour, B. Roynette, and P. Vallois, Explicit solutions of some fourth order partial differential equations via iterated Brownian motion, Seminar on Stochastic Analysis, Random Fields and Applications (Ascona, 1996), Progr. Probab., vol. 45, Birkhäuser, Basel, 1999, pp. 39-61.

[4] K. Burdzy and A. Mạdrecki, An asymptotically 4-stable process, The Journal of Fourier Analysis and Applications (1995), Special issue, 97-117.

[5] L. Debbi, On some properties of a higher fractional differential operator which is not in general selfadjoint, preprint, (2005). 
[6] L. Debbi and L. Abbaoui, Explicit solution of some fractional heat equations via Lévy motion, to appear in Maghreb Mathematical Review.

[7] L. Debbi and M. Dozzi, On the solution of non linear stochastic fractional partial differential equations, Stochastic Processes and Their Applications 115 (2005), no. 11, 1764-1781.

[8] W. Feller, On a generalization of Marcel Riesz' potentials and the semi-groups generated by them, Comm. Sém. Math. Univ. Lund [Medd. Lunds Univ. Mat. Sem.] 1952 (1952), Tome Supplementaire, $72-81$.

[9] T. Funaki, Probabilistic construction of the solution of some higher order parabolic differential equation, Proceedings Japan Academy Series A Mathematical Sciences 55 (1979), no. 5, 176-179.

[10] C. W. Gardiner, Handbook of Stochastic Methods. For Physics, Chemistry and the Natural Sciences, Springer Series in Synergetics, vol. 13, Springer, Berlin, 1983.

[11] R. Gorenflo and F. Mainardi, Random walk models for space-fractional diffusion processes, Fractional Calculus \& Applied Analysis 1 (1998), no. 2, 167-191.

[12] B. I. Henry and S. L. Wearne, Fractional reaction-diffusion, Phys. A 276 (2000), no. 3-4, 448-455.

[13] K. J. Hochberg, A signed measure on path space related to Wiener measure, The Annals of Probability 6 (1978), no. 3, 433-458.

[14] G. Jumarie, Complex-valued Wiener measure: an approach via random walk in the complex plane, Statistics \& Probability Letters 42 (1999), no. 1, 61-67.

[15] T. Komatsu, On the martingale problem for generators of stable processes with perturbations, Osaka Journal of Mathematics 21 (1984), no. 1, 113-132.

[16] V. J. Krylov, Some properties of the distribution corresponding to the equation $\partial u / \partial t=$ $(-1)^{q+1} \partial^{2 q} u / \partial x^{2 q}$, Soviet Mathematics Doklady 132 (1960), 1254-1257 (Russian), translated as Soviet Mathematics Doklady 1 (1960), 760-763.

[17] A. Le Méhauté, J. A. T. Machado, J. C. Trigeassou, and J. Sabatier, Fractional differentiation and its applications, Proceedings of the 1st IFAC Workshop on Fractional Differentiation and Its Applications (FDA '04), vol. 2004-1, ENSEIRB, Bordeaux, 2004, pp. 353-358.

[18] X. Leoncini and G. M. Zaslavsky, Jets, stickiness, and anomalous transport, Physical Review E. Statistical, Nonlinear, and Soft Matter Physics. 65 (2002), no. 4, 046216-1-046216-16.

[19] E. Lukacs, Characteristic Functions, Griffin's Statistical Monographs \& Courses, no. 5, Hafner, New York, 1960, 2nd edition 1970.

[20] F. Mainardi, Y. Luchko, and G. Pagnini, The fundamental solution of the space-time fractional diffusion equation, Fractional Calculus \& Applied Analysis 4 (2001), no. 2, 153-192.

[21] K. S. Miller and B. Ross, An Introduction to the Fractional Calculus and Fractional Differential Equations, John Wiley \& Sons, New York, 1993.

[22] T. Nakajima and S. Sato, On the joint distribution of the first hitting time and the first hitting place to the space-time wedge domain of a biharmonic pseudo process, Tokyo Journal of Mathematics. 22 (1999), no. 2, 399-413.

[23] Y. Nikitin and E. Orsingher, On sojourn distributions of processes related to some higher-order heat-type equations, Journal of Theoretical Probability 13 (2000), no. 4, 997-1012.

[24] K. Nishimoto, Fractional Calculus, Descartes Press, Koriyama, 1994.

[25] K. Nishioka, Stochastic calculus for a class of evolution equations, Japanese Journal of Mathematics. 11 (1985), no. 1, 59-102.

[26] _ A stochastic solution of a high order parabolic equation, Journal of the Mathematical Society of Japan. 39 (1987), no. 2, 209-231.

[27] _ Boundary conditions for one-dimensional biharmonic pseudo process, Electronic Journal of Probability. 6 (2001), no. 13, 1-27.

[28] S. G. Samko, A. A. Kilbas, and O. I. Marichev, Fractional Integrals and Derivatives. Theory and Applications, Gordon and Breach Science, Yverdon, 1993.

[29] G. Samorodnitsky and M. S. Taqqu, Stable Non-Gaussian Random Processes. Stochastic Models with Infinite Variance, Stochastic Modeling, Chapman \& Hall, New York, 1994. 


\section{Explicit solutions of some FPDEs via subordinators}

[30] K.-I. Sato, Lévy Processes and Infinitely Divisible Distributions, Cambridge Studies in Advanced Mathematics, vol. 68, Cambridge University Press, Cambridge, 1999.

[31] L. Sirovich, Techniques of Asymptotic Analysis, Applied Mathematical Sciences, vol. 2, Springer, New York, 1971.

[32] V. V. Uchaikin and V. M. Zolotarev, Chance and Stability. Stable Distributions and Their Applications, Modern Probability and Statistics, VSP, Utrecht, 1999.

Latifa Debbi: Institut Élie Cartan, Université Henri Poincaré - Nancy 1, B.P. 239,

54506 Vandoeuvre-lès-Nancy Cedex, France; Department of Mathematics, Faculty of Sciences, Ferhat Abbas University, El-Maabouda Sètif 19000, Algeria

E-mail address: debbi@iecn.u-nancy.fr

Current address: Department of Mathematics, Faculty of Sciences, University of M'sila, B.P. 166, Ichbilia, M'sila 28000, Algeria

E-mail address: ldebbi@yahoo.fr 


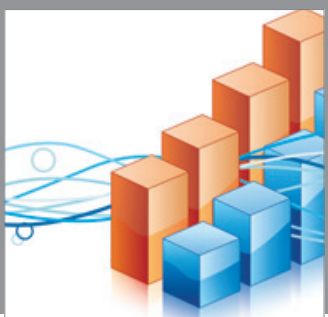

Advances in

Operations Research

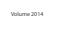

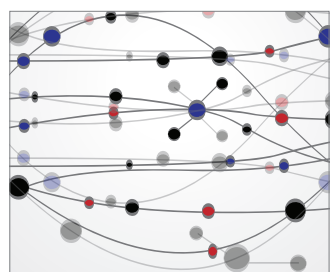

\section{The Scientific} World Journal
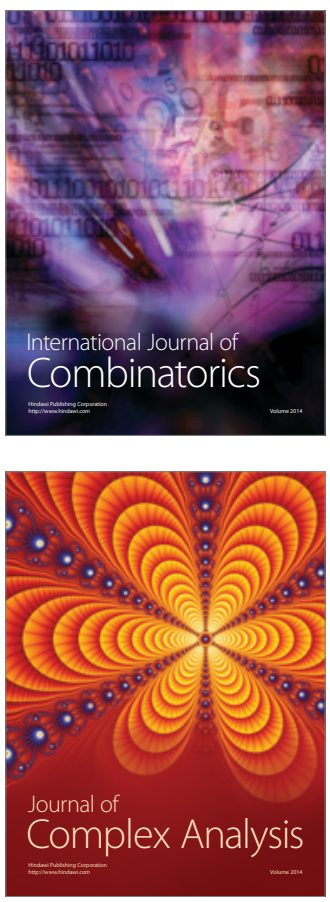

International Journal of

Mathematics and

Mathematical

Sciences
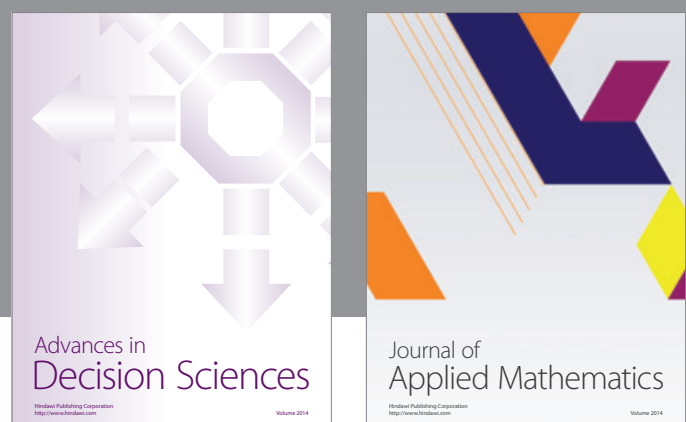

Journal of

Applied Mathematics
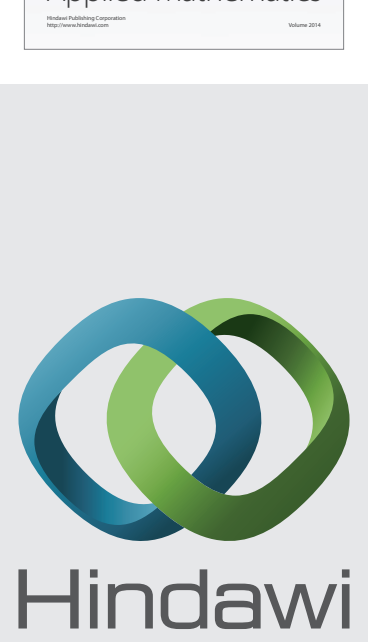

Submit your manuscripts at http://www.hindawi.com
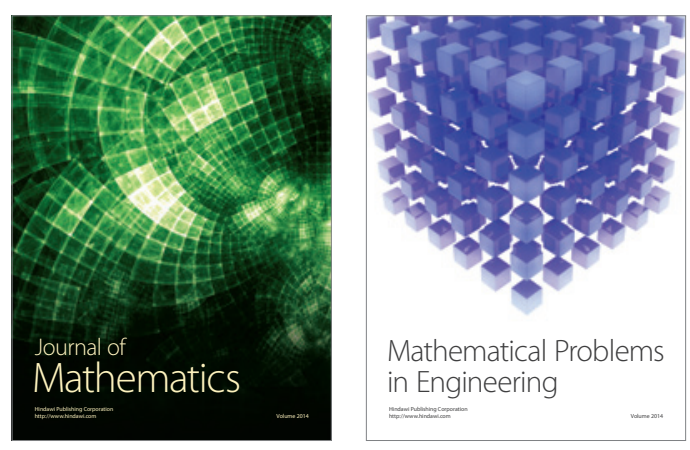

Mathematical Problems in Engineering
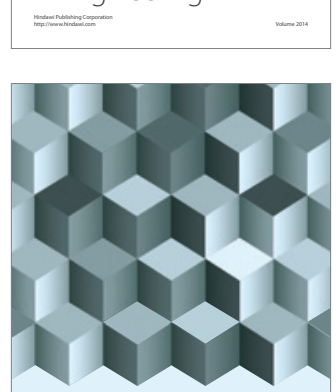

Journal of

Function Spaces
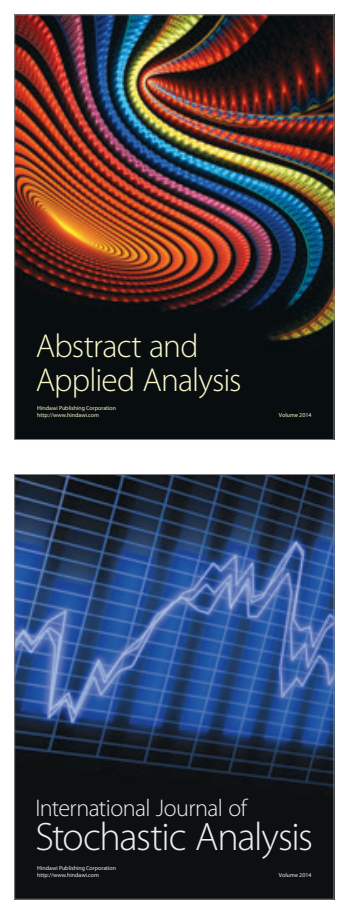

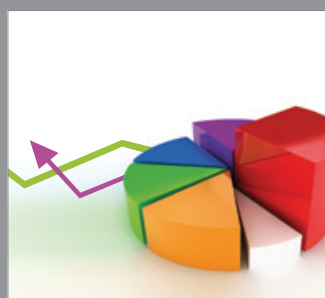

ournal of

Probability and Statistics

Promensencen
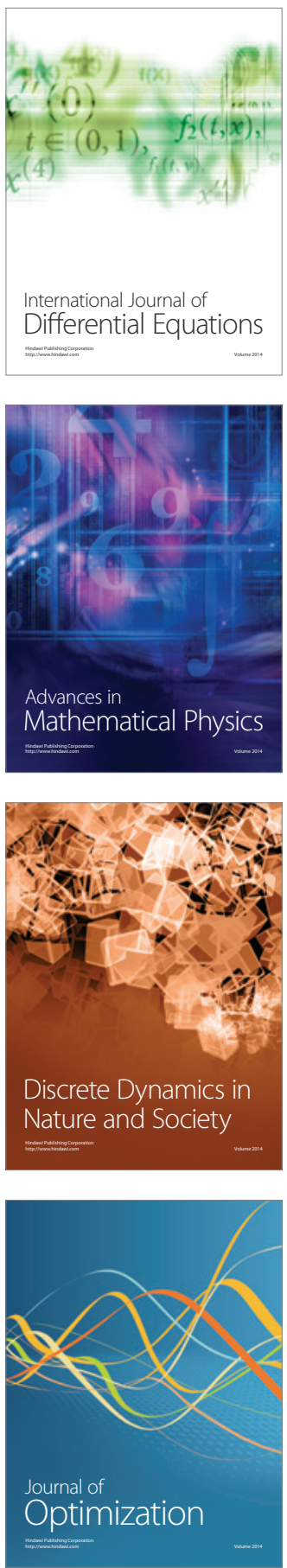